See discussions, stats, and author profiles for this publication at: https://www.researchgate.net/publication/230955451

\title{
Bi-Hamiltonian structures of d-Boussinesq and Benney-Lax equations
}

Article in Journal of Physics A General Physics - January 1999

DOI: 10.1088/0305-4470/27/1/013

2 authors, including:

2. Hasan Gümral

Yeditepe University

50 PUBLICATIONS 180 CITATIONS

SEE PROFILE 
Bi-Hamiltonian structures of d-Boussinesq and Benney-Lax equations

This article has been downloaded from IOPscience. Please scroll down to see the full text article.

1994 J. Phys. A: Math. Gen. 27193

(http://iopscience.iop.org/0305-4470/27/1/013)

View the table of contents for this issue, or go to the journal homepage for more

Download details:

IP Address: 144.122.42.186

The article was downloaded on 10/03/2011 at 05:44

Please note that terms and conditions apply. 


\title{
Bi-Hamiltonian structures of d-Boussinesq and Benney-Lax equations
}

\author{
H Gümral† and Y Nutkuł \\ $\uparrow$ Department of Physics, Tübitak, Marmara Research Centre, 41470, Gebze, Kocaeli, \\ Turkey \\ \$Department of Mathematics, Bilkent University, 06533, Ankara, Turkey
}

Received 3 April 1993, in final form 26 August 1993

\begin{abstract}
The dispersionless-Boussinesq and Benney-Lax equations are equations of hydrodynamic type which can be obtained as reductions of the dispersionless Kadomtsev-Petviashvili equation. We find that for the three-component reduction, the dispersionless Boussinesq and Benney-Lax equations are the same up to a diffeomorphism. This equivalence becomes manifest when the equations of motion are cast into the form of a triplet of conservation laws. Furthermore, in this form we are able to recognize a non-trivial scaling symmetry of these equations which plays an important role in the construction of their bi-Hamiltonian structure. We exhibit a pair of compatible Hamiltonian operators which belong to a restricted class of Dubrovin and Novikov operators appropriate to a system of conservation laws. The recursion operator for this system generates three infinite sequences of conserved Hamiltonians.
\end{abstract}

\section{Introduction}

The system of first-order quasi-linear evolution equations were called equations of hydrodynamic type by Dubrovin and Novikov [1] who found that the natural Hamiltonian structure of these equations is given by a first-order operator which is defined in terms of a flat Riemannian metric. Equations of hydrodynamic type quite often admit at least bi-Hamiltonian structure and are therefore completely integrable according to Magri's theorem [2]. The remarkable result that the geometry of the space of field variables plays a fundamental role in the definition of the Dubrovin-Novikov operator has attracted much attention. We refer to [3] for a survey of the Russian literature on this subject. The simplest systems that give rise to two-component equations of hydrodynamic type are classical quasi-linear secondorder wave equations associated with the names of Euler, Poisson and Chaplygin-Born-Infeld. The multi-Hamiltonian structure of these equations was given in [4-9]. For more than two components there are only a few examples of equations of hydrodynamic type, see Tsarev [10] for a list of physically interesting equations. Prominent among those is Zakharov's [11] multi-layered shallow water equations.

Mathematically, equations of hydrodynamic type appear naturally as reductions of the Zabolotskaya-Khokhlov ( $\mathrm{zK}$ ) equation, which is also referred to as the dispersionless Kadomtsev-Petviashvili (KP) equation. These reductions are due Zakharov [11], Gibbons and Kodama [12] and Kodama [13,14]. In this paper we shall be 
concerned with some three-component reductions of the $\mathrm{zK}$-equation and present their bi-Hamiltonian structure.

\section{Hamiltonian stuctures of hydrodynamic type}

Equations of hydrodynamic type consist of the system of first-order quasi-linear evolution equations

$$
u_{i}^{i}=v_{,}^{i}\left(u^{k}\right) u_{x}^{i} \quad i=1,2, \ldots, n
$$

and their multi-Hamiltonian structure is given by the Dubrovin-Novikov operator

$$
f^{i j}=g^{i j}(u) D-g^{i m} \Gamma_{m k}^{i}(u) u_{x}^{k}
$$

where $g^{i j}$ are the contravariant components of a Riemannian metric with vanishing curvature and $\Gamma_{j k}^{\prime}$ are the Christoffel symbols for the Levi-Civita connection compatible with this metric. $D$ denotes the total derivative with respect to $x$.

The Hamiltonian functional associated with this operator is defined by the zerothorder conserved densities of the system (1). They can be obtained as solutions of the system of second-order equations

$$
\partial_{i} \partial_{k} H v_{j}^{i}=\partial_{l} \partial_{l} H v_{k}^{i}
$$

which is an overdetermined system. We shall be concerned with equations of hydrodynamic type which are expressible in the form of conservation laws, that is the 1 -forms

$$
\sigma^{i}=v_{k}^{i} \mathrm{~d} u^{k}
$$

are closed, $\mathrm{d} \sigma^{i}=0$. If the given basis $\left\{\sigma^{i}\right\}$ is not closed, then we shall suppose that there exists a transformation $S_{k}^{i}$ such that

$$
\sigma \rightarrow S \cdot \sigma \quad v \rightarrow S \cdot v \cdot S^{-1}
$$

whereby the transformed basis 1 -forms are closed.

We shall consider a subclass of DN-type operators

$$
J=\left(\Gamma+\Gamma^{\prime}\right) D+\Gamma_{x}
$$

which are defined in terms of a single matrix $\Gamma$. This form of Hamiltonian operator, which we shall call Dubrovin-Novikov-Liouville-Poisson-type operators, is the one appropriate to a reformulation of (1) in terms of conserved quantities. There will be no confusion of the matrix $\Gamma$ with the Christoffel symbols which will not be used any further in this paper. The Hamiltonian flow appropriate to the operator (6) is given by

$$
\sigma=\left(\Gamma+\Gamma^{\prime}\right) \cdot d \nabla H+d \Gamma \cdot \nabla H
$$

where $\nabla=\left(\partial_{1}, \ldots, \partial_{n}\right)^{t}$ with respect to $u^{i}$ and

$$
\mathrm{d} \sigma=\mathrm{d} \Gamma^{\prime} \wedge \mathrm{d} \nabla H
$$

which vanishes for conservative systems.

Equations of hydrodynamic type which are in the form of conservation laws admit a first Hamiltonian structure with constant coefficients which can be brought to the form

$$
g_{i j}=\eta_{i j}=\delta_{1, n-1}
$$


without loss of generality. This is an involution

$$
\eta \cdot v \cdot \eta^{-1}=v^{t}
$$

for the conservative form of the equations of motion. The metric $\eta$ enters into the definition of the conserved quantity

$$
H_{0}=\eta_{u s} u^{i} u^{j}
$$

which usually corresponds to momentum.

When the entries of the matrix $\Gamma$ are expressed in terms of vectorial quantities appearing in its columns, (7) and (8) as well as the Jacobi identities and the compatibility condition can be written in a compact form. To be more specific, for $n=3$ we let

$$
\eta=\left(\begin{array}{lll}
0 & 0 & 1 \\
0 & 1 & 0 \\
1 & 0 & 0
\end{array}\right) \quad \Gamma=\left(\begin{array}{ccc}
p & q & r \\
k & m & l \\
f & g & h
\end{array}\right)
$$

and introduce the column vectors $\gamma^{\prime}$ of $\Gamma$ and $\tilde{\gamma}^{\prime}$ of $\Gamma^{\prime}$ with the components given according to (12) and

$$
\tilde{\gamma}^{1}=\left(\begin{array}{c}
p \\
q \\
r
\end{array}\right) \quad \tilde{\gamma}^{2}=\left(\begin{array}{c}
k \\
m \\
l
\end{array}\right) \quad \tilde{\gamma}^{3}=\left(\begin{array}{c}
f \\
g \\
h
\end{array}\right) .
$$

Then, from (8) we find the following nine equations

$$
\tilde{\gamma}_{v}^{2}=\tilde{\gamma}_{w}^{3} \quad \tilde{\gamma}_{v}^{1}=\bar{\gamma}_{u}^{3} \quad \tilde{\gamma}_{w}^{1}=\tilde{\gamma}_{u}^{2}
$$

For the elementary conserved quantity $H_{0}$.

The Jacobi identities are the coefficients of independent tri-vectors and they become simply

$$
\begin{aligned}
& \left(\left(\gamma^{[t}+\tilde{\gamma}^{[i}\right)^{t} \cdot \nabla\right) \tilde{\gamma}^{\prime]}=0 \\
& \left(\gamma_{x}^{(t}\right)^{t} \cdot \nabla \gamma^{j]}=0
\end{aligned}
$$

where square brackets denote anti-symmetrization. The same formulas apply to $n$-component DNLP-type operators as well.

\section{3. d-Boussinesq and Benney-Lax equations}

The dispersionless Boussinesq equations

$$
\left(\begin{array}{l}
w^{1} \\
w^{2} \\
w^{3}
\end{array}\right)_{t_{1}}=\left(\begin{array}{ccc}
0 & 1 & 0 \\
w^{1} & 0 & 1 \\
-w^{2} & -3 w^{1} & 0
\end{array}\right)\left(\begin{array}{l}
w^{1} \\
w^{2} \\
w^{3}
\end{array}\right)_{x}
$$

have been considered by Kodama [13] in connection with the reduction of the 
ZK- equation and were shown to admit the conserved densities

$$
\begin{aligned}
& H_{1}=w^{1} \quad H_{2}=w^{2} \quad H_{3}=w^{3}+\left(w^{1}\right)^{2} \\
& H_{4}=\left(w^{1}\right)^{3}+w^{1} w^{3}+\frac{1}{2}\left(w^{2}\right)^{2}=H_{1} H_{3}+\frac{1}{2} H_{2}^{2} \\
& H_{5}=\frac{1}{2}\left(w^{1}\right)^{2} w^{2}+w^{2} w^{3}=H_{2}\left(H_{3}-\frac{1}{2} H_{1}^{2}\right) \\
& \ldots
\end{aligned}
$$

which are infinite in number. In another context, Gibbons and Kodama [12] studied the Lax reduction of the Benney moment equations whose first flow is given by

$$
\left(\begin{array}{l}
v^{1} \\
v^{2} \\
v^{3}
\end{array}\right)_{t_{1}}=\left(\begin{array}{ccc}
0 & 1 & 0 \\
-2 v^{1} & 0 & 1 \\
-v^{2} & 0 & 0
\end{array}\right)\left(\begin{array}{l}
v^{1} \\
v^{2} \\
v^{3}
\end{array}\right)_{x}
$$

and they showed that it also admits an infinite sequence of conserved densities

$$
\begin{aligned}
& K_{1}=v^{1} \quad K_{2}=v^{2} \quad K_{3}=v^{3}-\frac{1}{2}\left(v^{1}\right)^{2} \quad K_{4}=0 \\
& K_{\mathrm{5}}=-\frac{1}{2}\left(v^{1}\right)^{3}+v^{1} v^{3}+\frac{1}{2}\left(v^{1}\right)^{2}=K_{1} K_{3}+\frac{1}{2} K_{2}^{2} \\
& K_{6}=-\left(v^{1}\right)^{2} v^{2}+v^{2} v^{3}=-\frac{1}{2} K_{1}^{2} K_{2}+K_{2} K_{3} \\
& K_{7}=-\frac{3}{2} v^{1}\left(v^{2}\right)^{2}+\frac{3}{2}\left(v^{3}\right)^{2}-\frac{3}{2}\left(v^{1}\right)^{2} v^{3}+\frac{5}{8}\left(v^{1}\right)^{4}=-\frac{3}{2} K_{1} K_{2}^{2}+\frac{3}{2} K_{3}^{2}+\frac{1}{4} K_{1}^{4} \\
& \ldots
\end{aligned}
$$

Equations (17) and (19) are not in conservative form as in both cases the third component of $\sigma$ is not closed. However, the Pfaff system $\left\{\sigma^{i}\right\}$ is an integrable one. For instance, in the case of (17) $\mathrm{d} \sigma^{1}=\mathrm{d} \sigma^{2}=0$ and

$$
\mathrm{d} \sigma^{3}=-2 \mathrm{~d} w^{1} \wedge \sigma^{1}
$$

which satisfies Frobenius' criterion for integrability. If we choose new coordinates from the infinite sequence of conserved densities

$$
(v, w, u)=\left(w^{1}, w^{2}, w^{3}+\left(w^{1}\right)^{2}\right)
$$

we find that (17) are cast into the form of conservation laws

$$
\left(\begin{array}{l}
v \\
w \\
u
\end{array}\right)_{t}=\left(\begin{array}{ccc}
0 & 1 & 0 \\
-v & 0 & 1 \\
-w & -v & 0
\end{array}\right)\left(\begin{array}{l}
v \\
w \\
u
\end{array}\right)_{x}
$$

There is also a similar transformation for the Benney-Lax equations that brings them to manifestly conservative form. This is given by

$$
(v, w, u)=\left(v^{1}, v^{2}, v^{3}-\frac{1}{2}\left(v^{1}\right)^{2}\right)
$$

and the result is again (22). Thus the Kodama reduction of the dispersionless Boussinesq equation (17) and the Lax reduction of the Benney moment equations (19) are equivalent. Henceforth we shall be primarily concerned with the system (22). The results for (17) and (19) can be obtained through the transformations (21) and (23).

\subsection{Bi-Hamiltonian structure}

Since the systems (17) and (19) can be put into a form of a triplet of conservation laws 
(22), it follows that they admit

$$
J_{0}^{i k}=\eta^{i k} D
$$

as first Hamiltonian operator. The Hamiltonian density appropriate to this operator is given by

$$
H_{1}^{\prime}=-\frac{1}{2} v^{2} w+u w
$$

which is the image of $H_{5}$ under the transformation (21).

The system (17) has no non-trivial scaling symmetry among the field variables whereas the fields in (22) as well as those of (19) can be assigned dimensions [9, 15]

$$
[v]=1 \quad[w]=\frac{3}{2} \quad[u]=2
$$

and this requires $[x]-[t]=\frac{1}{2}$. In the new variables, the conserved densities of the system (17) become

$$
v, w, u, u v+\frac{1}{2} w^{2}, u w-\frac{7}{2} w v^{2}, \ldots
$$

with dimension

$$
1, \frac{3}{2}, 2,3, \frac{7}{2}, \ldots
$$

which indicates that a conserved density with dimension $\frac{5}{2}$ is absent. This fact manifests itelf in a non-existence result for a second Hamiltonian structure with the momentum density (11). Although all our earlier results about the equations of hydrodynamic type use the momentum as the Hamiltonian density of the second structure, we have now an example where this is not so. The proper choice of the Hamiltonian density is a crucial step in the construction of the second Hamiltonian structure. We shall first construct the Hamiltonian operator by analysing further the equations (7) and (8), and then determine the appropriate Hamiltonian density.

For the second Hamiltonian stucture of (22) we find from (8) and (14) that

$$
\begin{array}{ll}
\tilde{\gamma}_{v}^{2}=-w \tilde{\gamma}_{u}^{1}-v \tilde{\gamma}_{w}^{1} & \tilde{\gamma}_{v}^{3}=-w \bar{\gamma}_{w}^{1}+v^{2} \tilde{\gamma}_{u}^{1} \\
\tilde{\gamma}_{w}^{2}=-w \tilde{\gamma}_{u}^{1}+\tilde{\gamma}_{v}^{1} & \tilde{\gamma}_{w}^{3}=-w \bar{\gamma}_{u}^{1}-v \bar{\gamma}_{w}^{1} \\
\tilde{\gamma}_{u}^{2}=\tilde{\gamma}_{w}^{1} & \tilde{\gamma}_{u}^{3}=\tilde{\gamma}_{v}^{1}
\end{array}
$$

while equations (7) reduce to

$$
\begin{aligned}
& 2 h=2 v^{2} p-w(k+q) \\
& g+l=2 w p-v(k+q) \\
& 2 m=f+r-2 v p .
\end{aligned}
$$

It turns out that (28) and the relations (29) are necessary and sufficient for the satisfaction of the equations (15) and (16) resulting from the Jacobi identity.

The integrability conditions of (28) give three vector equations

$$
\begin{aligned}
& \tilde{\gamma}_{v w}^{1}=-w \tilde{\gamma}_{u u}^{1}-v \tilde{\gamma}_{u w}^{1} \\
& \tilde{\gamma}_{v v}^{1}=-w \tilde{\gamma}_{u w}^{1}+v^{2} \tilde{\gamma}_{u u}^{1} \\
& \tilde{\gamma}_{w w}^{1}=-v \tilde{\gamma}_{u u}^{1}+\tilde{\gamma}_{u v}^{1}
\end{aligned}
$$

which when written in component form are simply the equations (3) satisfied by the zeroth-order conserved densities. Thus the functins $p, q$ and $r$ must be elements of the 
sequence (27) of conserved densities. The proper choice of the entries of the first row of $\Gamma$ can be done most conveniently by analysing the dimensions of the elements in the second Hamiltonian operator. We find that for (22)

$$
\Gamma=\left(\begin{array}{ccc}
v & 2 w & 3 u \\
w & 2 u-v^{2} & -3 v w \\
u & -2 v w & v^{3}-\frac{3}{2} w^{2}
\end{array}\right)
$$

and the resultng DNLP-type Hamiltonian operator (6) is given by

$$
J=\left(\begin{array}{ccc}
2 v & 3 w & 4 u \\
3 w & 4 u-2 v^{2} & -5 v w \\
4 u & -5 v w & 2 v^{3}-3 w^{2}
\end{array}\right) D+\left(\begin{array}{ccc}
v & 2 w & 3 u \\
w & 2 u-v^{2} & -3 v w \\
u & -2 v w & v^{3}-\frac{3}{2} w^{2}
\end{array}\right)
$$

Thus we have the second Hamiltonian structure. The Hamiltonian density that yields the equations of motion (22) for the second Hamiltonian operator is $w / 2$.

The Hamiltonian operator (32) satisfies the Jacobi identities. This can be verified by checking that (15) and (16) are satisfied for (31). There is, however, an alternative proof which is based on the theorem of Dubrovin and Novikov. From the expression $\Gamma+\Gamma^{\gamma}$ we can read off the contravariant components of a metric which should be flat if the Jacobi identities are satisfied. Inverting it we find that the metric is given by

$$
\begin{aligned}
\mathrm{d} s^{2}=\Delta^{-1}\left[\left(8 u v^{3}-12 u w^{2}-4 v^{5}-19 v^{2} w^{2}\right) \mathrm{d} v^{2}+\left(8 u v-4 v^{3}-9 w^{2}\right) \mathrm{d} u^{2}\right. \\
+ \\
+2\left(-8 u^{2}+2 v^{4}-3 v w^{2}\right) \mathrm{d} w^{2}+2\left(-16 u^{2}+8 u v^{2}-15 v w^{2}\right) \mathrm{d} u \mathrm{~d} v \\
\left.+2 w\left(-20 u v-6 v^{3}+9 w^{2}\right) \mathrm{d} v \mathrm{~d} w-4 w\left(6 u+5 v^{2}\right) \mathrm{d} u \mathrm{~d} w\right]
\end{aligned}
$$

where

$$
\Delta=-64 u^{3}+32 u^{2} v^{2}+16 u v^{4}-144 u v w^{2}-8 v^{6}-56 v^{3} w^{2}+27 w^{4}
$$

is the inverse of the determinant of the metric. It can be verified directly that all the components of the Riemann tensor vanish identically for the metric (33). The recursion operator

$$
R=\left(\Gamma+\Gamma^{t}\right) \cdot \eta+\Gamma_{x} \cdot \eta D^{-1}
$$

divides the sequence (27) into three infinite sequences

$$
\begin{gathered}
v \rightarrow u v+\frac{1}{2} w^{2} \rightarrow \frac{5}{2} 1 u^{2} v+\frac{5}{2} w^{2} u-\frac{5}{2} v^{2} w^{2}-\frac{1}{4} v^{5} \\
\frac{1}{2} w \rightarrow u w-\frac{1}{2} w v^{2} \rightarrow 3 w u^{2}-3 u w v^{2}+\frac{3}{4} w v^{4}-2 v w^{3} \\
u \rightarrow \frac{3}{2} u^{2}-\frac{3}{2} v w^{2}+\frac{1}{4} v^{4} \rightarrow \frac{7}{2} u^{3}+\frac{7}{4} u v^{4}-\frac{7}{8} w^{4}+\frac{7}{3} v^{3} w^{2}-\frac{21}{2} u v w^{2}
\end{gathered}
$$

because its action is confined to any one of them. As in all equations of hydrodynamic type, the existence of an operator that will carry a conserved quantity in one sequence into an element of another sequence remains an open question. We note that once again $\partial / \partial u$ plays the role of the inverse of the recursion operator. Finally, there is no conserved quantity with dimension $(1+4 n) / 2, n=1,2, \ldots$ in any of the above seqeunces. The missing density with dimension $\frac{5}{2}$ is only the first of its kind.

The bi-Hamiltonian structure of the dispersionless Boussinesq equation (17) can be obtained using the inverse transformation (21). The first Hamiltonian operator 
becomes

$$
J_{0}^{\prime}=\left(\begin{array}{ccc}
0 & 0 & D \\
0 & D & 0 \\
D & 0 & -2\left(w^{1} D+D w^{1}\right)
\end{array}\right)
$$

with the Hamiltonian function $H_{5}$, and the second Hamiltonian operator is given by

$$
\begin{aligned}
J^{\prime}= & \left(\begin{array}{ccc}
2 w^{1} & 3 w^{2} & 4 w^{3} \\
3 w^{2} & 2\left(2 w^{3}+\left(w^{1}\right)^{2}\right) & -11 w^{1} w^{2} \\
4 w^{3} & -11 w^{1} w^{2} & -16 w^{1} w^{3}-3\left(w^{2}\right)^{2}-6\left(w^{1}\right)^{3}
\end{array}\right) D \\
& +\left(\begin{array}{ccc}
w_{x}^{1} & 2 w_{x}^{2} & 3 w_{x}^{3} \\
w_{x}^{2} & \left(2 w^{3}+\left(w^{1}\right)^{2}\right)_{x} & -5 w^{1} w_{x}^{2}-9 w^{2} w_{x}^{1} \\
w_{x}^{3} & -6 w^{1} w_{x}^{2}-2 w^{2} w_{x}^{1} & -\left(3\left(w^{1}\right)^{3}+\frac{3}{2}\left(w^{2}\right)^{2}+8 w^{1} w^{3}\right)_{x}
\end{array}\right)
\end{aligned}
$$

and the corresponding Hamiltonian density is $w^{2} / 2$. Note that the first operator is of DNLP-type, that is, it can be written on the form of $J_{1}$ in (6), whereas the second one is not. This is another indication that DNLP opertors are natural for the conservative form of the equations of motion.

\section{Conclusions}

One of the techniques for studying equations of hydrodynamic type consists in the use of Riemann invariants as dependent variables. This is a powerful method because the matrix $v_{k}^{i}$ is then diagonalized. It has yielded many interesting results [3]. An alternative approach starts with the equations of motion written in the form of conservation laws. The variables that enter into such a formulation are often the physical variables. We have shown that in terms of the physical variables the Hamiltonian operator is expressible in the DNLP form (6) which is a reduction of the Dubrovin-Novikov operator. This approach is also useful because it manifests a scaling symmetry of the equations of motion which carries over into the elements of the second Hamiltonian operator. Its virtue lies in the fact that it does not require us to guess the correct Hamiltonian density before the Hamiltonian operator is available. The example of (22) is very instructive in this regard.

\section{Acknowledgments}

This work was in part supported by The Turkish Scientific Research Council TÜBI'TAK under TBAG-CG-1.

\section{References}

[1] Dubrovin B A and Novikov S P 1983 Sov. Math. Dokl. 27665

[2] Magri F 1978 J. Math. Phys. 191356

[3] Dubrovin B A and Novikov S P 1989 Russian Mathematical Surveys 4435

[4] Nutku Y 1983 J. Phys. A: Math. and Gen. 164195

[5] Neyzi F and Nutku Y 1987 J. Math. Phys. 281499 
[6] Nutku Y 1987 J. Math. Phys. 282579

[7] Olver P J and Nutku Y $1988 J$. Math. Phys. 291610

[8] Arik M, Neyzi F, Nutku Y, Olver P J and Verosky J M 1989 J. Math. Phys. 301338

[9] Gümral H and Nutku Y 1990 J. Math. Phys. 312606

[10] Tsarev S P 1991 Proceeding of NATO-ASI'Singular Limits of Dispersive Waves' Lyon

[11] Zakharov V E 1980 Func. Anal. Appl. 14

[12] Gibbons J and Kodama Y Proceeding of International Workshop 'Nonlinear and Turbulent Processes in Physics' Kiev

[13] Kodama Y 1988 Prog. Theor. Phys. Suppl. 94184

[14] Kodama Y 1988 Nonlinear Evolution Equations ed J P Leon (Singapore: World Scientific) p 97

[15] Gümral H $1992 J_{.}$Phys. A: Math. and Gen. 255141 